\title{
In der Offenen Jugendarbeit geht noch was
}

\section{Demokratische Selbstorganisation von benachteiligten Kindern und Jugendlichen}

Die Coronakrise brachte es ans Licht: Offene Kinder- und Jugendarbeit (OKJA) hat sich sehr lange kaum um die Förderung von Selbstorganisation gekümmert. Das gilt besonders in Bezug auf ihre größte Besucher_innengruppe: benachteiligte Kinder und Jugendliche. Da geht aber noch mehr.

$\mathrm{I}$ n Zeiten der Corona-Lockdowns mussten die Fachkräfte der offenen Jugendeinrichtungen überall sein, um überhaupt Kontakt zu Kindern und Jugendlichen zu halten: Im digitalen Sozialraum, im physischen Sozialraum und in der Einrichtung unter Sicherheitsbedingungen (Deinet und Sturzenhecker 2021). Das ließ sich gar nicht gleichzeitig bewältigen, ohne die Kinder und Jugendlichen selbst in die Organisation der Jugendarbeit mit einzubeziehen. Plötzlich wurde klar, dass sie über viel größere Fähigkeiten der Nutzung digitaler/ sozialer Medien verfügen als die Fachkräfte und man war auf ihre Unterstützung angewiesen. Ihre Erfahrung,

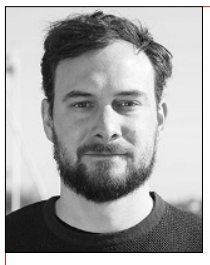

Moritz Schwerthelm

Universität Hamburg, Hamburg, Deutschland

*1986; Erziehungs- und Bildungswissenschaften, M.A., wissenschaftlicher Mitarbeiter am Arbeitsbereich Sozialpädagogik der Fakultät für Erziehungswissenschaft, Universität Hamburg. moritz.schwerthelm@uni-hamburg.de

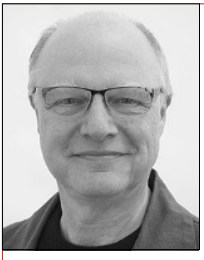

Benedikt Sturzenhecker

Universität Hamburg, Hamburg, Deutschland

*1958; Dipl. Päd., Dr. phil., Univ.-Prof. für Sozialpädagogik und außerschulische Bildung an der Fakultät für Erziehungswissenschaft der Universität Hamburg.

benedikt.sturzenhecker@uni-hamburg.de

Zusammenfassung Die Assistenz von Selbstorganisation benachteiligter junger Menschen ist eine zentrale fachliche Aufgabe Offener Kinder- und Jugendarbeit. Sie entsteht aus den strukturellen Potenzialen der OKJA für demokratische Partizipation. Das Konzept von Selbstorganisation in der Jugendarbeit ist von einer Selbstorganisation neoliberaler Lesart abzugrenzen. Hemmnisse professioneller Assistenz von Selbstorganisation werden analysiert und Ansätze zu einer besseren Förderung entwickelt.

Schlüsselwörter Offene Kinder- und Jugendarbeit, Selbstorganisation, Partizipation, Demokratiebildung über diese Medien untereinander Kontakt zu halten und sich zu organisieren, half auch, Aktionen der Jugendarbeit im physischen Sozialraum zu ermöglichen. Obwohl die Fachkräfte es in den meisten Fällen gewohnt waren, Rahmen und Angebote der OKJA selbst zu organisieren und zu bestimmen, erzwang die Krise, die Potenziale der Partizipation und der Selbstorganisation der Ziel- und Besucher_innengruppen wieder zu entdecken. Hinzu kam, dass die noch 2020 erschienenen Untersuchungen zur Situation der Kinder und Jugendlichen in der Coronazeit (Andresen et al. 2020) deutlich machten, dass ein Großteil der befragten jungen Menschen kritisierte, zum fremdbestimmten Objekt von Erwachsenen und Politik gemacht sowie für das Ausbildungssystems funktionalisiert zu werden. Im Widerspruch dazu fordern sie politische Partizipation, mindestens zu Fragen des Umgangs mit Corona; aus ihrer Sicht und Betroffenheit.

Der Bedarf, die Forderung und die fachliche Aufgabe, Partizipation und Selbstorganisation von Kindern und Jugendlichen auch in der OKJA stärker zu ermöglichen, wird auch nach dem Abebben der Pandemie unabweisbar bleiben. Hinzu kommt hier, dass die OKJA überwiegend benachteiligte Kinder und Jugendliche erreicht. Diese sind aber in der öffentlichen Debatte über die aktuelle Selbstorganisation von Jugendlichen (einerseits die „Fridays“ und andererseits Formen rechtspopulistischer und rechtsextremer Selbstorganisation) kaum Thema. Wie so häufig werden ihnen die benachteiligenden Lebensverhältnisse von Armut, Bildungsungleichheit, Migrationsbedingungen, Diskriminierung und Marginalisierung etc. als ihr Defizit zugeschrieben und ihre Praxen, Stärken und Potenziale von Selbstorganisation werden verkannt. Im Folgenden wird gezeigt, dass sich die Aufgabe der Förderung von Selbstorganisation benachteiligter Kinder und Jugendlichen konzeptionell ohnehin stellt, was hier unter Selbstorganisation (nicht) verstanden werden soll, und wo eine Förderung in der Praxis ansetzen könnte. 


\section{Extrablick: Interessenvertretung in der Kinder- und Jugendhilfe}

\section{Selbstorganisation als fachlicher Auftrag Offener Kinder- und Jugendarbeit?}

Neuere organisationstheoretische Analysen beschreiben für die OKJA eine Hybridität hinsichtlich ihrer pädagogischen Funktionen der Kontrolle, Hilfe, (Demokratie-)Bildung und Selbstorganisation (Schwerthelm 2021). Der OKJA werden, neben ihren jugendarbeitstheoretisch formulierten Funktionen der sozialpädagogischen Ermöglichung von Demokratiebildung und Selbstorganisation (entsprechend des $\mathbb{1} 11$ und 12 SGB VIII) auch sozialarbeiterische Funktionen der Kontrolle und Hilfe zugewiesen, letztere besonders von Politik, Trägern, aber auch Fachkräften selbst. In der Alltagspraxis können diese Funktionen nicht eindeutig voneinander getrennt werden: Sie weisen Bezüge auf, überlappen sich und fordern von Fachkräften wie Besucher_innen ständige Wechsel von Rollen und Adressierungen, je nachdem, welcher der pädagogischen Funktionsaspekte gerade zum Thema wird. Zudem können all diese Funktionen - aufgrund der strukturellen Offenheit und Freiwilligkeit der OKJA - ohnehin nur verfolgt werden, wenn die Teilnehmenden mindestens passiv zustimmen oder aktiv die pädagogischen Settings mitgestalten. Hier entdecken wir ein strukturelles demokratisches Potenzial, dem die OKJA in Kombination mit ihrem gesetzlichen Auftrag zur Subjekt- und Demokratiebildung nach $\mathbb{1 1}$ SGB VIII, ihre Möglichkeiten zu Förderung demokratischer Partizipation und Demokratiebildung verdankt.

Die Selbstorganisationsfunktion sehen wir darin, Kindern und Jugendlichen ihre „soziale [...] Integration auf dem Wege der Mitwirkung an den prinzipiell demokratisch geregelten Handlungsbereichen der Zivilgesellschaft" (Richter et al. 2016, S. 108) zu ermöglichen. Dieser Blick auf Selbstorganisation stellt damit weniger Assistenz von Bildung als pädagogisches Handlungsziel in den Mittelpunkt, sondern das konkrete demokratische Handeln der Kinder und Jugendlichen. Aus dieser Perspektive besteht die Aufgabe von Jugendarbeiter_innen darin, gemeinsam mit Kindern und Jugendlichen demokratische Strukturen, Verfahren und Gremien in Einrichtungen und Angeboten zu entwickeln, mithilfe derer die gemeinsame Lebensführung und Umsetzung der differenten Interessen in den Einrichtungen selbst geregelt werden, aber auch Übergänge in kommunalpolitische Aushandlungsprozesse herzustellen. Das setzt voraus, sich konsequent an den Interessen und Themen der Adressat_innen zu orientieren und deren Aneignungs- und Handlungsweisen als relevant für die Gestaltung der OKJA selbst, ebenso wie der Gesellschaft insgesamt anzuerkennen und sie mit ihnen zu realisieren. Eine solche politische Bildung führt aber stringent von der demokratischen Partizipation zur demokratischen Selbstorganisation, wie es auch die „Partizipationsleitern" entwerfen.

\section{Zum Begriff von Selbstorganisation in der Jugend- arbeit}

Den traditionellen Begriff jugendarbeiterischer Selbstorganisation muss man gegen eine neoliberale Vorstellung von Selbstorganisation als Selbststeuerung des Individuums (Graefe 2019, S. 81 ff.) abgrenzen. Stefanie Graefe zeigt, dass Selbstorganisation in neoliberaler Lesart (SO-n) als Selbststeuerung des Individuums verstanden wird, während sich das jugendarbeiterische Konzept von Selbstorganisation (SO-j) auf Gruppen bezieht, die ihre Interessen so selbständig wie nur irgend möglich umsetzen sollen. SO-n zielt auf ein möglichst reibungsloses Funktionieren der Person angesichts gesellschaftlicher Anforderungen und Krisen. SO-j sucht eine kritisch-aktive Mitwirkung in der demokratischen Gesellschaft, indem Kinder und Jugendliche (auch organisiert in Initiativen, Vereinen und Verbänden), ihrer Themen in demokratische Konflikt- und Entscheidungsprozesse einbringen. SO-n fantasiert eine Autarkie der Person, die sich doch so aktiv an die Verhältnisse anpasst. SO-j hingegen sieht die soziale und gesellschaftliche Eingebundenheit der Kinder und Jugendlichen und damit die Notwendigkeit von Kooperation. Statt um eine Selbstregulation wie im Modell der SO-n, geht es für eine SO-j um eine kollektive Selbstverwaltung und demokratische Partizipation. SO-n zielt auf reinen Selbsterhalt, SO-j auf eine Realisierung von Eigensinn, die aber stets den Bezug auf das Gemeinwohl herstellt. Dahinter stehen auch unterschiedliche Freiheitskonzepte. Mit der SO-n ginge es um individuelle Freiheit, als darum, dass jede_r macht, was er/sie will. Mit SO-j geht es darum, sich mit seinen Interessen in ein Verhältnis zu anderen Gesellschaftsmitgliedern und ihren Interessen sowie den Institutionen zu setzen: es geht um soziale Freiheit.

\section{Hemmnisse von Selbstorganisation in der OKJA}

Selbstorganisation scheint sowohl in der Fachpraxis als auch in Konzepten der OKJA in den vergangenen Jahrzehnten wenig berücksichtigt worden zu sein, obwohl es fachliche wie historische Gründe gibt, sie in das Zentrum des Handelns zu stellen. Denn die OKJA weist seit der Jugendzentrumsbewegung deutliche Bezüge zum Konzept von Selbstorganisation auf (Templin 2015). Die selbstverwalteten Jugendräume machen immerhin zwischen vier und $20 \%$ der Einrichtungen der OKJA aus (Drumm et al. 2021). Vielerorts stehen diese „selbstorganisierte[n] Jugendräume [...] nicht nur in 
der Tradition der Reaktion auf fehlende Freizeitangebote, sondern sind - zumindest zum Teil - aus dem Bedürfnis Jugendlicher entstanden, sich von erwachsenendominierten, vorgefertigten Angeboten abzusetzen und Orte zu schaffen, die den eigenen, lebensphasenspezifischen Bedürfnissen und Interessen entsprechen " (Peucker et al. 2021, S. 489).

Mit der Dominanz der Hauptamtlichkeit in der OKJA scheint die fachliche Aufgabe der Unterstützung von Selbstorganisation jedoch in den Hintergrund getreten zu sein. Doch was hemmt die professionelle Förderung von Selbstorganisation? Schon allein die Struktur von Hauptamtlichkeit birgt die Gefahr, Selbstorganisation zu behindern, denn es ist immer schon jemand da, der/ die für die Organisation zuständig ist. Und dann gibt es die Immobilie, das „unbewegliche“ Jugendhaus, das offengehalten und gemanagt werden will. Selbstorganisation kann aber nicht an ein Haus gebunden werden, sondern muss auch darüber hinausgehen können. Fachkräfte können jedoch allein zeitlich kaum „draußen“ Selbstorganisation von Gruppierungen unterstützen. Aufgrund der strukturellen Offenheit und Freiwilligkeit der OKJA erfahren die Besucher_innen dort selten eine Verbindlichkeit und Kontinuität bzgl. ihres Engagements für die gemeinsamen Interessen; nicht nur, weil die Fachkräfte dafür zuständig sind, sondern auch, weil sie selbst kommen und gehen wie sie wollen. Aus dieser Unverbindlichkeit Selbstorganisation zu entwickeln, die ohne die Übernahme von Verantwortung nicht auskommt, fällt nicht leicht. Wenn man eigene Interessen selbst organisiert umsetzen will, muss man sich verbindlich verbinden und eine gewisse gegenseitige Verpflichtung für die gemeinsame Sache entwickeln. Damit stellt sich dann auch irgendwann die Frage der Gründung eines eigenen Vereins, der eine demokratische Struktur für die Binnen- und Außenverhältnisse zur Verfügung stellt. Damit entsteht ein Übergang zwischen OKJA und Jugendverbandsarbeit ( $\mathbb{1 2}$ SGB VIII).

Fachkräften fällt es aber wohl selten leicht, die Bildung einer Organisation zu unterstützen, die ihrer Einrichtung vermeintlich Konkurrenz macht. Damit ist auch das Problem des Legitimationsdrucks von Fachkräften in der OKJA angesprochen. Aus der Marginalität und ständigen öffentlichen Hinterfragung des Handlungsfeldes entsteht häufig der Wunsch, die Bedeutung und Leistung der eigenen pädagogischen Arbeit nachzuweisen. Fachkräfte wollen belegen, dass sie die Organisatoren sind, d. h. aber eben auch: nicht die Kinder und Jugendlichen. Selbstorganisation wird aus dieser Sicht überflüssig, weil die Fachkräfte schon alles so wunderbar anbieten, was die Kids benötigen. Häufig meinen sie deshalb die Bedürftigkeit der Zielgruppen nach Ange- boten, aber auch nach Hilfe und unter Umständen gar nach Prävention und Kontrolle, öffentlich demonstrieren zu müssen. Damit schreiben sie dann den Kindern und Jugendlichen Defizite zu und sich selbst die Funktion, diese zu beheben. Die Fachkräfte adressieren dann allerdings ihre Zielgruppen (und gerade die benachteiligten) als Objekte ihrer Hilfe, statt als Subjekte demokratischer Partizipation und Selbstorganisation.

\section{Ansätze einer Förderung von Selbstorganisation in der OKJA}

Gerade die benachteiligten Lebenslagen vieler Besucher_innen der OKJA verleiten die Fachkräfte dazu, besonders die Defizite dieser Kinder und Jugendlichen wahrzunehmen. Damit geschieht zweierlei:

1. Die Fachkräfte verkennen die Fähigkeiten und Leistungen von Selbstorganisation, über die diese Zielgruppen schon verfügen und

2. sie schwächen die Kinder und Jugendlichen noch, indem sie diese als Objekte von Hilfe und Kontrolle thematisieren.

Deren prekäre Lebensverhältnisse führen allerdings de facto zu einem großen Ausmaß von Selbstorganisation. Untersuchungen wie die von Klatt und Walter (2011) zeigen, dass benachteiligte Gruppen ihren problematischen Alltag sehr wohl selbstorganisiert bewältigen, durch die Pflege von privaten Freundes- und Familiennetzwerken, durch gegenseitige Hilfe, durch Identifikation mit ihrem Viertel und gekonnte Umgang mit knappen Ressourcen (ähnlich Calmbach und Borgstedt 2012; Sturzenhecker 2021 [2015]). Sie nehmen dies allerdings kaum selbst als Engagement und Leistung der Selbstorganisation wahr. Ihnen fehlt häufig das „Selbstzutrauen“ (Klatt und Walter 2011, S. 193), das Wissen und die spezifischen Ressourcen, ihre Selbstorganisation in die Bürgergesellschaft einzubringen. Gerade dort ist dann doch eine pädagogische Unterstützung gefragt: „In diesen Fällen wäre Anleitung oder - besser noch Hilfe zu Selbstorganisation notwendig " (Klatt und Walter 2011, S. 198). Diese kann und muss allerdings bei der Erkenntnis und Stärkung der schon vorhandenen Selbstorganisationsweisen der Kinder und Jugendlichen ansetzen. Eine Assistenz von Selbstorganisation wird nur gelingen, wenn sie die Adressat_innen nicht zunächst zu Objekten macht, sondern deren Fähigkeiten respektiert und im Dialog mit ihnen ausweitet.

Die Methode zur Förderung gesellschaftlich-demokratischen Engagements von Benachteiligten in der OKJA (Sturzenhecker und Schwerthelm 2021 [2015]) zeigt, wie durch Beobachtung und Dialog zunächst mit Kindern und Jugendlichen geklärt werden kann, was sie 


\section{Extrablick: Interessenvertretung in der Kinder- und Jugendhilfe}

wirklich betrifft und welche Interessen sie haben. Diese Themen mit ihnen durch demokratische Partizipation umzusetzen, eröffnet, Inhalte und Potenziale der Selbstorganisation zu erkennen und Schritt für Schritt auszubauen. Das reicht von Interessengruppen in Einrichtungen und Angeboten, die Teilbereiche (mal auch nur Projekte) selbstorganisieren, über selbstorganisierte Gruppen, die Räumlichkeiten und Ressourcen der Einrichtung nutzen (mit unterschiedlich starker Begleitung durch Hauptamt), bis zu Gruppen Jugendlicher, deren eigenständige Aktivitäten im Sozialraum, von Fachkräften besucht und unterstützt werden. Hierzu könnten Fachkräfte zunächst überprüfen, welche Interessenbzw. selbstorganisierten Gruppen sie in Einrichtung und Sozialraum bereits kennen, aber bisher nicht als solche wahrgenommen haben (für methodische Anleitungen siehe Schwerthelm 2020, S. 34 ff). Dabei sollte auch immer angestrebt werden, noch mehr Selbstorganisation zu ermöglichen, zum Beispiel in Form von selbstverwalteten Jugendräumen und Treffs, die zunächst noch von Fachkräften begleitet werden (Drumm et al. 2021), sich in eigenen Vereinen organisieren und schließlich autonome Einrichtungen ohne Hauptamt betreiben.

Dieser ideale Prozess beginnt ganz klein im Alltag durch die Beobachtung des Handelns der Kinder und Jugendlichen und durch die Wahrnehmung ihrer schon vorhandenen Praxen von Selbstorganisation. Ein solcher Prozess könnte modellhaft wie folgt gestaltet werden:

1. Beobachtung der Selbstorganisation der Kinder und Jugendlichen und Anerkennung dieser Tätigkeiten;

2. Auswertung des beobachteten Handelns in Bezug auf jene Interessen, die mindestens zwei Besucher innen teilen;

3. (medial unterstützte) Resonanz auf diese Themen und dialogische Klärung mit den Beteiligten, wie eine Umsetzung organisiert werden könnte;

4. Einbezug anderer Interessierter bzw. Betroffener;

5. demokratische Selbstbestimmung bzgl. der Arbeitsweisen der Gruppe;

6. Integration der Gruppe in den demokratischen Mitbestimmungsprozess der Gestaltung der Einrichtung insgesamt (ggf. Übergänge in Vollversammlung, Jugendrat o. ä.);

7. Abklärung mit den Interessen anderer Gruppierungen;

8. Organisation von mehr Wissen und Ressourcen zur Umsetzung des Themas;

9. zunehmende Ausweitung der Selbstorganisation in und außerhalb der Einrichtung.
Dabei folgt man der Maxime: Soviel Selbstbestimmung und Selbstorganisation wie irgend möglich, so viel Unterstützung wie gerade noch nötig. Wie weit diese Prozesse einen solchen idealen Weg annehmen, ist nicht relevant. Es geht stattdessen darum, überhaupt die Potenziale von Selbstorganisation benachteiligter Kinder und Jugendlichen in der OKJA zu erkennen, anerkennend aufzugreifen und Schritt für Schritt auszuweiten. Statt sie als Objekte von sozialarbeiterischer Hilfe und Kontrolle zu behandeln, gilt es, die demokratische Partizipation und Selbstorganisation der benachteiligten Kinder und Jugendlichen zu stärken.

Eingegangen. 15. Juni 2021

Angenommen. 20. Juli 2021

Funding. Open Access funding enabled and organized by Projekt DEAL.

Open Access. Dieser Artikel wird unter der Creative Commons Namensnennung 4.0 International Lizenz veröffentlicht, welche die Nutzung, Vervielfältigung, Bearbeitung, Verbreitung und Wiedergabe in jeglichem Medium und Format erlaubt, sofern Sie den/die ursprünglichen Autor(en) und die Quelle ordnungsgemäß nennen, einen Link zur Creative Commons Lizenz beifügen und angeben, ob Änderungen vorgenommen wurden.

Die in diesem Artikel enthaltenen Bilder und sonstiges Drittmaterial unterliegen ebenfalls der genannten Creative Commons Lizenz, sofern sich aus der Abbildungslegende nichts anderes ergibt. Sofern das betreffende Material nicht unter der genannten Creative Commons Lizenz steht und die betreffende Handlung nicht nach gesetzlichen Vorschriften erlaubt ist, ist für die oben aufgeführten Weiterverwendungen des Materials die Einwilligung des jeweiligen Rechteinhabers einzuholen.

Weitere Details zur Lizenz entnehmen Sie bitte der Lizenzinformation auf http://creativecommons.org/licenses/by/4.0/deed.de.

\section{Literatur}

Andresen, S., Lips, A., Möller, R., Rusack, T., Schröer, W., Thomas, S., \& Wilmes, J. (2020). Erfahrungen und Perspektiven von jungen Menschen während der Corona-Maßnahmen. Erste Ergebnisse der bundesweiten Studie JuCo. Hildesheim: Universitätsverlag Hildesheim.

Calmbach, M., \& Borgstedt, S. (2012). „Unsichtbares“ Politikprogramm? Themenwelten und politisches Interesse von „bildungsfernen“ Jugendlichen. In W. Kohl \& A. Seibring (Hrsg.), „Unsichtbares" Politikprogramm? Themenwelten und politisches Interesse von bildungsfernen Jugendlichen. Schriftenreihe der Bundeszentrale für politische Bildung. Bonn: Bundeszentrale für politische Bildung.

Deinet, U., \& Sturzenhecker, B. (2021). Neustart der Offenen Kinder- und Jugendarbeit in NRW in der Corona-Zeit - Zusammenfassung der Ergebnisse, Bewertung und Empfehlungen. https://www.ew.uni-hamburg.de/einrichtungen/ew2/sozialpaedagogik/forschung/neustart-der-offenen-kinder-und-jugendarbeit-in-nrw-waehrend-der-corona-krise.html. Zugegriffen: 16. Mai 2021.

Drumm, T., Groß, A., \& Koch, T. (2021). Selbstverwaltete Jugendeinrichtungen. In U. Deinet, B. Sturzenhecker, L. von Schwanenflügel \& M. Schwerthelm (Hrsg.), Handbuch Offenen Kinder- und Jugendarbeit (5. Aufl. S. 583-595). Wiesbaden: VS.

Graefe, S. (2019). Resilienz im Krisenkapitalismus. Wider das Lob der Anpassungsfähigkeit. Bielefeld: transcipt. 
Klatt, J., \& Walter, F. (2011). Entbehrliche der Bürgergesellschaft? Sozial Benachteiligte und Engagement. Bielefeld: transcript.

Peucker, C., Pluto, L., \& van Santen, E. (2021). Empirisches Wissen zu Typen und Merkmalen von Einrichtungen der Offenen Kinder- und Jugendarbeit. In U. Deinet, B. Sturzenhecker, L. von Schwanenflügel \& M. Schwerthelm (Hrsg.), Handbuch Offenen Kinder- und Jugendarbeit (5. Aufl. S. 479-493). Wiesbaden: VS.

Richter, E., Richter, H., Sturzenhecker, B., Lehmann, T., \& Schwerthelm, M. (2016). Bildung zur Demokratie - Operationalisierung des Demokratiebegriffs für pädagogische Institutionen. In Raingard Knauer \& B. Sturzenhecker (Hrsg.), Demokratische Partizipation von Kindern (S. 107-129). Weinheim: BeltzJuventa.

Schwerthelm, M. (2021). Offene Kinder- und Jugendarbeit als hybride Organisation - ein Modell zur Diskussion ihrer Funktionen. In U. Deinet, B. Sturzenhecker, L. von Schwanenflügel \& M. Schwerthelm (Hrsg.), Handbuch Offenen Kinder- und Jugendarbeit (5. Aufl. S. 883-908). Wiesbaden: VS.

Schwerthelm, M. (2020). Partizipation in der Offenen Jugendarbeit in Luxemburg. Konzeptionelle Grundlagen, methodische Anleitungen und Qualitätsstandards. Service National de la Jeunesse (Hrsg.). Luxemburg. https:// www.enfancejeunesse.lu/de/2007032 brochure snj_partizipation-in-der-offenen-jugendarbeit_al_web.Zugegriffen: 18. Mai 2021.

Sturzenhecker, B. (2021). Konzeptionelle Grundlagen für die Offene Kinder- und Jugendarbeit. Unter Mitarbeit von Moritz Schwerthelm (4. Aufl.). Gesellschaftliches Engagement von Benachteiligten fördern, Bd. 1. Gütersloh: Bertelsmann Stiftung.

Sturzenhecker, B., \& Schwerthelm, M. (2021). Methodische Anregungen und Praxisbeispiele für die Offene Kinder-und Jugendarbeit (4. Aufl.). Gesellschaftliches Engagement von Benachteiligten fördern, Bd. 2. Gütersloh: Bertelsmann Stiftung.

Templin, D. (2015). Freizeit ohne Kontrollen. Die Jugendzentrumsbewegung in der Bundesrepublik der 1970er-Jahre. Göttingen: Wallstein.

Hier steht eine Anzeige.

Springer 